\title{
UTILIZAÇÃO DE FARINHA MISTA DE AVEIA E TRIGO NA ELABORAÇÃO DE BOLOS
}

\author{
JOÃO TOMAZ DA SILVA BORGES* \\ MONICA RIBEIRO PIROZI** \\ SUZANA MARIA DELLA LUCIA ${ }^{* * *}$ \\ POLLYANNA CARDOSO PEREIRA*** \\ ALLAN ROBLEDO FIALHO E MORAES**** \\ VANESSA CRISTINA CASTRO***
}

\begin{abstract}
O objetivo do presente trabalho foi avaliar a viabilidade tecnológica e sensorial do uso da farinha de aveia em mistura com farinha de trigo $(0,15,30 \mathrm{e}$ $45 \%$ de adição) na elaboração de bolos. A farinha de aveia apresentou maior granulometria que a de trigo, de modo que maior quantidade dessa farinha nas formulações eleva a retenção de partículas nas peneiras utilizadas. Foi observado pela análise farinográfica que a absorção de água e o tempo necessário para formação de massa homogênea e estável às condições de mistura aumentaram com o incremento no teor de farinha de aveia. A densidade das massas cruas aumentou com a adição da farinha de aveia, sendo o oposto observado para o volume do bolo. A análise sensorial revelou que as formulações com $0 \%$ e $30 \%$ de farinha de aveia foram as mais aceitas pelos consumidores. $O$ emprego de $30 \%$ de farinha de aveia não modificou a aceitação dos atributos sabor, textura e impressão global do bolo, sendo viável a utilização dessa porcentagem para substituição parcial da farinha de trigo na elaboração de bolos.
\end{abstract}

PALAVRAS-CHAVE: FARINHA DE AVEIA; BOLO; FARINHA MISTA; FARINHA DE TRIGO.

* Doutorando em Ciência e Tecnologia de Alimentos, Departamento de Tecnologia de Alimentos (DTA), Universidade Federal de Viçosa (UFV), Viçosa, MG (e-mail: jtsborges@yahoo.com.br).

** Professora, Doutora em Ciência de Cereais e Panificação, DTA/UFV, MG (e-mail: mpirozi@ufv.br).

*** Doutorandas em Ciência e Tecnologia de Alimentos, DTA/UFV, Viçosa, MG (e-mails: smdlucia@yahoo.com.br; pollycardoso@yahoo.com.br; vanessariso@yahoo.com.br).

**** Graduando do curso de Engenharia de Alimentos, UFV, MG (e-mail: allanrfm@yahoo.com.br).

B.CEPPA, Curitiba, v. 24, n. 1, p. 145-162, jan./jun. 2006 


\section{INTRODUÇÃO}

A aveia (Avena sativa L.) constitui cereal de excelente valor nutricional. Destaca-se entre os cereais por fornecer aporte energético e nutricional equilibrado, por conter em sua composição química aminoácidos, ácidos graxos, vitaminas e sais minerais indispensáveis ao organismo humano e, principalmente, pela composição de fibras alimentares (9\% a 11\%). Apresenta teor protéico variando de 12,4\% a $24,5 \%$ no grão descascado e teor de lipídios entre 3,1\% a 10,9\%, distribuídos pelo grão composto, predominantemente, de ácidos graxos insaturados (PEDÓ e SGARBIERI, 1997; SÁ et al., 2000; WEBSTER, 1986; WEBER, GUTKOSKI, ELIAS, 2002).

O consumo de farinha e farelo de aveia é benéfico para a saúde humana em razão da elevada concentração de fibras. A fibra alimentar pode ser classificada em solúvel e insolúvel em água. A fibra solúvel da aveia compõe-se de pectinas, B-glucanas, mucilagens, algumas hemiceluloses e amido resistente. Os principais componentes das fibras insolúveis são a celulose e as hemiceluloses (PETERSON, 1992; GUTKOSKI e TROMBETTA, 1999). WOOD, WEIZ e BLACKWELL (1991) e SÁ et al. (2000) afirmaram que as B-glucanas, moléculas lineares compostas de ligações glicosídicas $\beta-1,3$ e ß-1,4, estão presentes em grande quantidade na aveia (concentrações de $3,9 \% \mathrm{e}$ $6,8 \%$ na cariopse e de $5,8 \%$ e $8,9 \%$ no farelo).

Os efeitos fisiológicos das fibras dietéticas no metabolismo dos lipídios têm sido amplamente investigados e a maioria dos estudos ressalta as propriedades hipocolesterolêmicas das fibras solúveis. As evidências indicam que o consumo de 3-15 g/dia de diversas fibras solúveis, incluindo guar, pectina, farelo de aveia e fibra de soja reduzem os níveis de colesterol e glicose no sangue em torno de 5 a $15 \%$ (ROY, VEJA-LOPEZ e FERNANDEZ, 2000; GRIZARD, DALLE e BARTHOMEUF, 2001).

Estudos têm revelado propriedades tecnológicas, sensoriais, nutricionais e funcionais vantajosas quanto à utilização da aveia na alimentação humana. Seus produtos podem ser utilizados como ingredientes na panificação devido às suas excelentes propriedades de absorção de umidade, o que retarda o envelhecimento de pães, 
bolos e biscoitos. Além disso, seus derivados têm habilidade de estabilizar componentes lipídicos em razão de suas propriedades antioxidantes. Trata-se do único cereal cuja proteína apresenta balanço de aminoácidos relevantes sob o ponto de vista nutricional, bem como teor protéico superior ao dos demais cereais (LOCKART e HURT, 1986; HOSENEY, 1990; McKECHNIE, 1983, citado por GUTKOSKI e PEDÓ, 2000; TEDRUS et al., 2001; SEABRA et al., 2002).

Grande parte dos produtos de panificação é composta por ingredientes que desempenham funções específicas no processo de formação da massa. Embora os constituintes possam variar em grau de importância no processo de fabricação, todos exercem determinada função. Muitas vezes, a maior ou menor importância desses ingredientes está associada com a quantidade adicionada à massa e ao tipo de produto.

Na década de 60, a utilização de farinhas mistas tinha como objetivo a substituição parcial da farinha de trigo para redução das importações desse cereal. Depois, as pesquisas com farinhas mistas foram direcionadas para a melhoria da qualidade nutricional de produtos alimentícios e para suprir a necessidade dos consumidores por produtos diversificados (TIBURCIO, 2000). Vários fatores devem ser considerados na utilização de farinhas mistas para produção de alimentos. As características das farinhas sucedâneas devem reduzir ao máximo os efeitos da substituição para se obter alimentos com cor aceitável, sabor agradável e boa textura (BARBOSA, 2002).

Mudanças no processamento e a crescente exigência do consumidor por alimentos com qualidade sensorial, nutricional e que tragam benefícios à saúde incentivam o estudo de novos ingredientes para a indústria de alimentos (IDRIS et al., 1996; MOSCATTO, PRUDENCIOFERREIRA e HAULY, 2004).

Entre os produtos de panificação, o bolo vem adquirindo crescente importância no que se refere ao consumo e comercialização no Brasil. O desenvolvimento tecnológico possibilitou mudanças nas indústrias transformando a produção de pequena para grande escala (MOSCATO, PRUDÊNCIO-FERREIRA E HAULY, 2004). Embora não constitua alimento básico como o pão, o bolo é aceito e consumido por pessoas de qualquer idade. Trata-se de produto obtido pela mistura, 
homogeneização e cozimento conveniente de massa preparada com farinhas, fermentadas ou não e outras substâncias alimentícias (como, por exemplo, leite, ovos e gordura). A farinha de trigo constitui o principal componente das formulações por fornecer a matriz em torno da qual os demais ingredientes são misturados para formar a massa (ELDASH e CAMARGO, 1982).

O objetivo do presente trabalho foi avaliar a viabilidade tecnológica e sensorial do uso da farinha integral de aveia em mistura com farinha de trigo na elaboração de bolos.

\section{MATERIAL E MÉTODOS}

O presente trabalho foi realizado no Laboratório de Amido e Farinha do Departamento de Tecnologia de Alimentos, da Universidade Federal de Viçosa (UFV).

Empregou-se delineamento de blocos completos casualizados na montagem do experimento, conforme OTT (1993), correspondendo os níveis $(0 \%, 15 \%, 30 \%, 45 \%)$ de farinha de aveia na formulação ao tratamento e blocos aos dias de produção dos bolos.

\subsection{MATÉRIA-PRIMA E FARINHAS MISTAS (FM)}

Foram utilizados farinha de trigo especial, farinha integral de aveia, fermento químico, açúcar cristal, ovos in natura, leite pasteurizado e margarina de marcas comerciais, adquiridos no comércio local (Viçosa).

Misturou-se a farinha de aveia integral à farinha de trigo nas porcentagens de 0, 15 (FM 1), 30 (FM 2) e 45 (FM 3) de substituição.

\subsection{CLASSIFICAÇÃO GRANULOMÉTRICA}

Foram peneiradas $100 \mathrm{~g}$ de cada amostra de farinha de aveia, farinha de trigo e farinhas mistas, durante 10 minutos. Usou-se conjunto de seis peneiras arredondadas, vibratórias e com aberturas das malhas variando de 30, 40, 45, 50, 60 a 70 ABNT. Em seguida, as quantidades retidas em cada peneira foram pesadas e expressas em percentagens. 
Com maior homogeneidade no tamanho de partículas, as farinhas de aveia e trigo foram misturadas nas respectivas formulações para obtenção da farinha mista de aveia e trigo.

\subsection{FARINOGRAFIA}

Utilizou-se farinógrafo Brabender e o método 54-21 da AACC (1983) para a farinografia, obtendo-se inicialmente a curva de titulação e, posteriormente, o farinograma completo.

\subsection{PREPARO DO BOLO}

Misturou-se o ovo, o açúcar e a margarina por 1 minuto em batedeira até formação de mistura homogênea. Adicionou-se a farinha e o leite, batendo por 2 minutos. Ao final, acrescentou-se o fermento e a massa foi batida por mais 1 minuto. Colocou-se a massa em forma, previamente forrada com papel manteiga, levando-a ao forno pré-aquecido a $180^{\circ} \mathrm{C}$. O tempo de assamento foi de, aproximadamente, 40 minutos. $\mathrm{Na}$ Tabela 1 constam os ingredientes utilizados nas diferentes formulações.

\section{TABELA 1 - INGREDIENTES UTILIZADOS NAS DIFERENTES FORMULAÇÕES}

\begin{tabular}{lcccc}
\hline \multirow{2}{*}{ Ingredientes } & \multicolumn{4}{c}{ Formulações* $^{*}$} \\
\cline { 2 - 5 } & (0\%aveia) & $\mathbf{1 ( 1 5 \% a v e i a )}$ & 2(30\%aveia) & 3(45\%aveia) \\
\hline Farinhade Trigo & 100,0 & 85,0 & 70,0 & 55,0 \\
Farinha Aveia & 0,0 & 15,0 & 30,0 & 45,0 \\
Açícar & 76,7 & 76,7 & 76,7 & 76,7 \\
Ovo & 40,0 & 40,0 & 40,0 & 40,0 \\
Margarina & 20,0 & 20,0 & 20,0 & 20,0 \\
Fermento & 3,3 & 3,3 & 3,3 & 3,3 \\
Leite & 93,3 & 93,3 & 93,3 & 93,3 \\
\hline
\end{tabular}

*Porcentagem dos ingredientes em relação a $100 \%$ do peso total da farinha mista. 


\subsection{ANÁLISE DE DENSIDADE DA MASSA CRUA}

Calculou-se a densidade da massa formulada, proveniente das diferentes misturas, dividindo a quantidade da massa (em gramas) presente em béquer pelo volume desse (PATON, LAROCQUE e HOLME, 1981). O volume padronizado para o presente estudo foi de $400 \mathrm{~mL}$.

\subsection{ANÁLISES FÍSICAS DO BOLO}

Os bolos foram submetidos à avaliação de volume, contorno e simetria de acordo com o método 10-90 da AACC (2000).

Para os dados de densidade da massa e de volume do bolo utilizou-se a análise de variância, seguida pelo teste de Duncan $(\alpha=0,05)$ para comparação de médias (OTT, 1993).

\subsection{ANÁLISE SENSORIAL}

Realizou-se o teste de aceitação no Laboratório de Análise Sensorial do Departamento de Tecnologia de Alimentos da UFV em cabines individuais.

Quarenta e sete julgadores não-treinados (consumidores), com idade entre 16 e 36 anos, sendo $77 \%$ mulheres participaram do teste. Residentes da cidade de Viçosa foram selecionados para efetuar a análise com base no hábito de consumir bolo.

As amostras das quatro formulações de bolo foram avaliadas quanto aos atributos sabor, textura e impressão global, mediante escala hedônica estruturada de nove pontos (STONE e SIDEL, 1993; ANZALDÚA-MORALES, 1994). As amostras foram servidas monadicamente de acordo com delineamento de blocos completos casualizados. Os dados, obtidos por meio de ficha de avaliação (Figura 1) foram submetidos à análise de variância e ao teste de Duncan $(\alpha=0,05)$ para comparação de médias (OTT, 1993).

Para as análises estatísticas utilizou-se o pacote estatístico SAS (1985). 
FIGURA 1 - MODELO DE FICHA DE RESPOSTA PARA O TESTE DE ACEITAÇÃO USANDO ESCALA HEDÔNICA

\begin{tabular}{|c|c|c|c|c|}
\hline Nome: & Sexo: & Idade: & \multicolumn{2}{|c|}{ Data: 1} \\
\hline \multicolumn{5}{|c|}{$\begin{array}{l}\text { Por favor, prove a amostra e avalie as características àdireita de acordo com a escala } \\
\text { abaixo: }\end{array}$} \\
\hline \multicolumn{5}{|c|}{ Código da amostra: } \\
\hline 9. Gostei extremamente & & Atributos: & & Nota \\
\hline 8- Gostei muito & & Sabor: & & \\
\hline 7. Gostei moderadamente & & Textura: & & \\
\hline 6- Gostei ligeiramente & & Impressẫo g & lobal: & \\
\hline 5- Indiferente & & & & \\
\hline 4- Desgostei ligeiramente & & & & \\
\hline 3- Desgostei moderadamente & & & & \\
\hline 2- Desgostei muito & & & & \\
\hline 1. Desgostei extremamente & & & & \\
\hline \multicolumn{5}{|l|}{ Comentários: } \\
\hline
\end{tabular}

\section{RESULTADOS E DISCUSSÃO}

\subsection{DISTRIBUIÇÃO DO TAMANHO DE PARTÍCULAS DAS FARINHAS DE AVEIA, DE TRIGO E MISTAS}

A característica granulométrica da matéria-prima constitui aspecto relevante na elaboração de massas alimentícias e produtos de padaria, pois a distribuição adequada de partículas permite maior uniformidade do produto elaborado.

A granulometria influencia diretamente a capacidade de absorção de água, as características sensoriais (como aparência, sabor e textura) e o tempo de cozimento das massas alimentícias (LINDEN e LORIENT, 1994; BORGES et al., 2003). A Figura 2 apresenta a análise granulométrica das farinhas de aveia, de trigo e mistas (FM 1, FM 2, FM 3) utilizadas na elaboração dos bolos. 
A farinha de aveia apresentou maior granulometria quando comparada com a farinha de trigo. Entretanto, esses resultados encontram-se de acordo com os dados relatados por GUTKOSKI e PEDÓ (2000). Notouse que com o aumento de farinha de aveia na formulação houve incremento na retenção de partículas nas peneiras com abertura de 30, 40, 50, 60 e 70 ABNT.

\section{FIGURA 2 - DISTRIBUIÇÃO (\%) DO TAMANHO DE PARTÍCULAS DA FARINHA DE AVEIA, FARINHA DE TRIGO E FARINHAS MISTAS (FM 1, FM 2, FM 3)}

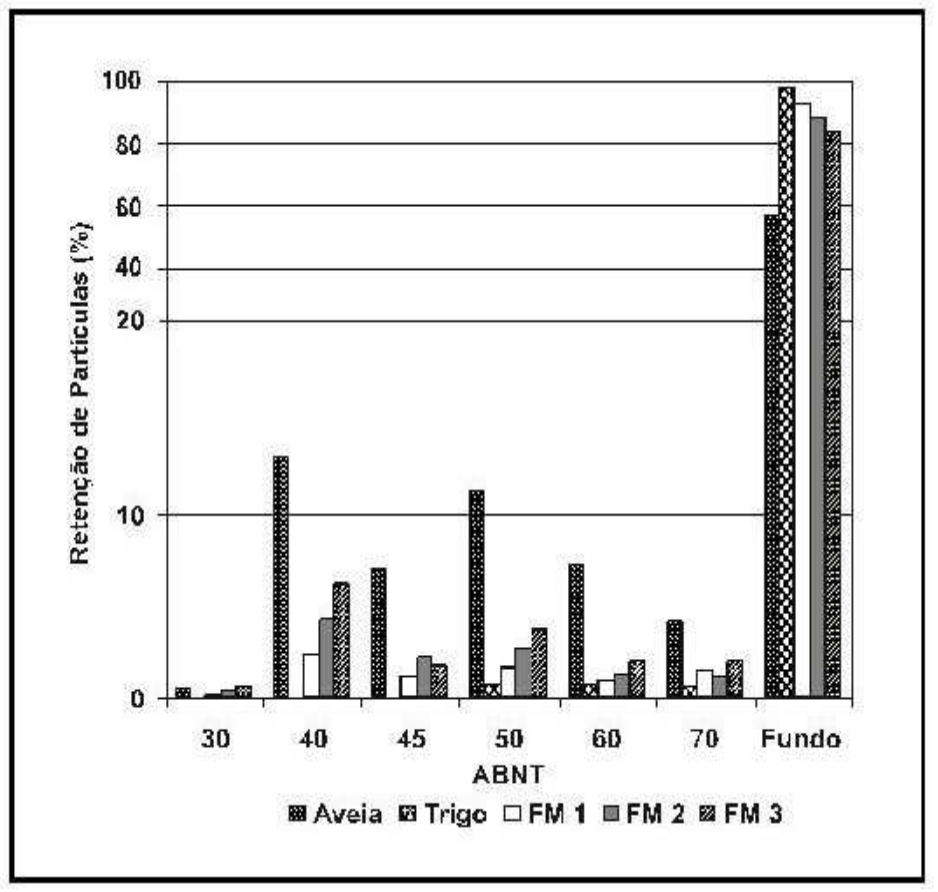

Percentual acima de $98 \%$ da farinha de trigo passou pela peneira 60 ABNT $(0,250 \mathrm{~mm})$ conforme determina a Portaria n. 354, de 18 de julho de 1996, o qual reduziu-se com o aumento de farinha de aveia na formulação (ANVISA, 2006).

A influência da granulometria de farinhas nas propriedades de bolos ainda não foi totalmente elucidada, embora seja aceito que partículas finas e uniformes promovem maior dispersibilidade da farinha na massa. 
Para PYLER (1988), granulometria extremamente fina da farinha não é sinônimo de qualidade. Teores elevados dessas partículas nas misturas podem prejudicar a estrutura interna (miolo) de produtos de panificação, tornando-a úmida e gomosa.

A principal exigência para a formação de massa adequada, dependendo do tipo de bolo, é que a mistura tenha quantidade suficiente de proteínas da clara de ovo para que durante o forneamento a estrutura protéica formada possa se espalhar sobre os componentes da farinha (MORR, HOFFMANN e BUCHHEIM, 2003). Caso contrário, a fraca estrutura protéica diminuirá a retenção de gás na massa e favorecerá a formação de estrutura compacta e baixo volume.

IWUOHA e NWAKANMA (1998) constataram que o tamanho da partícula e a concentração de pasta são fatores que influenciam, significativamente, a viscosidade de pasta, a densidade e a textura de produtos a base de carboidratos.

\subsection{ANÁLISE FARINOGRÁFICA}

Foram determinados os seguintes parâmetros: absorção de água (AA), tempo de chegada (TC), estabilidade (ES), tempo de desenvolvimento da massa (TDM) e índice de tolerância à mistura (ITM).

Os parâmetros farinográficos da farinha de trigo e das misturas estão apresentados na Tabela 2.

TABELA 2 - ABSORÇÃO DE ÁGUA E PROPRIEDADES DE MISTURA DA MASSA DE FARINHA DE TRIGO E FARINHAS MISTAS

\begin{tabular}{lccccc}
\hline Matérias-primas & $\begin{array}{c}\text { AA } \\
(\%)\end{array}$ & $\begin{array}{c}\text { TC } \\
(\mathbf{m i n})\end{array}$ & $\begin{array}{c}\text { ES } \\
(\mathbf{m i n})\end{array}$ & $\begin{array}{c}\text { TDM } \\
(\mathbf{m i n})\end{array}$ & $\begin{array}{c}\text { ITM } \\
\text { (U.F.) }\end{array}$ \\
\hline Farinha de trigo & 53,3 & 0,20 & 15,90 & 11,45 & 40 \\
FM 1 (15\% aveia) & 58,3 & 1,5 & 15,70 & 10,50 & 45 \\
FM 2 (30\% aveia) & 64,6 & 2,2 & 15,00 & 8,30 & 50 \\
FM 3 (45\% aveia) & 72,0 & 6,50 & 3,50 & 8,00 & 80 \\
\hline
\end{tabular}

$\mathrm{AA}=$ absorção de água; $\mathrm{TC}=$ tempo de chegada $\mathrm{ES}=$ estabilidade $; \mathrm{TDM}=$ tempo de desenvolvimento da massa; ITM = índice de tolerância à mistura. 
Durante o amassamento, as proteínas absorvem quantidade considerável de água e interagem para a formação da rede de glúten. De acordo com a composição físico-química apresentada pela farinha ocorre nessa etapa a formação de sistema coloidal complexo (envolvendo lipídios, amidos, açúcares, minerais e proteínas, dentre outros), que é responsável pelas características viscoelásticas necessárias para a produção de massas alimentícias (GUTKOSKI e JACOBSEN NETO, 2002).

Notou-se (Tabela 2) que a capacidade de absorção de água aumentou conforme o incremento do teor de farinha de aveia na mistura, o qual se deve principalmente ao conteúdo de fibras presentes na farinha de aveia (TEDRUS et al., 2001). Resultados semelhantes foram encontrados por TEDRUS, ORMENESE e SPERANZA (2001). Produtos de aveia são usados como ingredientes na panificação pela excelente capacidade de absorção de água, o que contribui para aumentar o rendimento e retardar o envelhecimento, além de atuar como estabilizante de componentes lipídicos por suas propriedades antioxidantes (GUTKOSKI e PEDÓ, 2000).

Vários autores têm demonstrado que conforme o aumento no conteúdo de proteína, a curva farinográfica atinge valores mais elevados. As frações que apresentam o mesmo teor protéico, mas que são de diferentes classes de farinha não produzem a mesma qualidade de proteína. GUTKOSKI et al. (1993) estudaram a viabilidade do uso de farinha de aveia em mistura com a farinha de trigo na elaboração de pães. Observaram que os teores de cinza, proteínas e lipídios aumentam com a elevação dos níveis de farinha de aveia à mistura, embora ocorra decréscimo de carboidratos.

O tempo de chegada (tempo em minutos desde o início até o ponto em que a curva alcança a linha das 500 unidades farinográficas) aumentou gradativamente à medida que se elevou a concentração de aveia na mistura. Esse comportamento sugere influência da fibra presente na farinha de aveia na formação da rede de glúten.

Observou-se diminuição nos valores da estabilidade conforme maiores porcentagens de farinha de aveia foram adicionadas à mistura, sendo menor variação obtida nas concentrações de $15 \%$ e $30 \%$. Houve redução mais acentuada da estabilidade na mistura com $45 \%$, 
indicando que o aumento no teor de farinha de aveia reduziu a força da massa. Isto sugere, segundo afirma BARBOSA (2002), diminuição na resistência das massas à fermentação e menor tolerância ao amassamento ou à ação mecânica.

O tempo de desenvolvimento da massa (TDM) indica a qualidade da proteína e farinhas fortes normalmente requerem maior tempo de desenvolvimento que farinhas fracas (PYLER, 1988; QUAGLIA, 1991). As três concentrações de misturas de farinhas apresentaram menor TDM em comparação com a farinha de trigo pura. Tais valores decresceram com o aumento da porcentagem de farinha de aveia, 0 que é característico de farinhas fracas, favoráveis à produção de bolos. A adição de farinha de aveia torna o glúten mais tenaz e menos elástico, alterando as propriedades reológicas da mistura já que suas proteínas não formam glúten (GUTKOSKI e PEDÓ, 2000).

Maiores valores do índice de tolerância à mistura (ITM) foram obtidos com o aumento da concentração de farinha de aveia, indicando o enfraquecimento da mistura para a formação de glúten. Comumente, farinhas que apresentam boa tolerância à mistura revelam menor ITM (maior índice de tolerância indica farinha mais fraca). Segundo GUTKOSKI e PEDÓ (2000) esse comportamento ocorre, provavelmente, pelo maior teor de lipídios, bem como pelo enfraquecimento do glúten proporcionado pela adição de farinha de aveia.

Ao utilizar farinhas mistas ocorrem alterações nas propriedades físicas e tecnológicas, que podem ser favoráveis ou não conforme o produto. No caso do bolo, a farinha fraca proporcionada pela mistura é desejável. A estrutura desse produto é mais leve e porosa, portanto depende menos de glúten do que produtos como pão.

No Brasil, a farinha empregada na produção de bolos é a mesma usada para fazer pães. EL-DASH e GERMANI (1994) recomendam o enfraquecimento dessa farinha forte pela adição de outras farinhas, como a farinha de aveia utilizada no presente estudo.

\subsection{ANÁLISES FÍSICAS}

A formulação contendo $30 \%$ de aveia não diferiu significativamente 
das formulações com $15 \%$ e $45 \%$ em relação à densidade da massa crua, mas todas diferiram da formulação com $0 \%$ de aveia (Tabela 3 ). Conforme afirma PYLER (1988), a densidade constitui medida de incorporação e retenção de ar na massa de bolo. Menor densidade significa maior incorporação de ar, conforme pode ser constatado na comparação entre a densidade da massa e a medida de volume do bolo. Valores de densidade altos (formulação com $45 \%$ de farinha de aveia) desfavorecem o volume do produto e provocam a formação de miolo fechado, massudo e de mau aspecto.

TABELA 3 - AVALIAÇÃO DE DENSIDADE DA MASSA CRUA, VOLUME, CONTORNO E SIMETRIA DO BOLO

\begin{tabular}{ccccc}
\hline Formulação & $\begin{array}{c}\text { Densidade da } \\
\text { massa crua }\end{array}$ & $\begin{array}{c}\text { Volume } \\
(\mathbf{m m})\end{array}$ & $\begin{array}{c}\text { Contorno } \\
(\mathbf{m m})\end{array}$ & $\begin{array}{c}\text { Simetria } \\
(\mathbf{m m})\end{array}$ \\
\hline (0\% aveia) & $0,93 \mathrm{c}$ & $144,7 \mathrm{a}$ & 4,3 & 0,0 \\
1 (15\% aveia) & $0,97 \mathrm{~b}$ & $122,7 \mathrm{~b}$ & 19,3 & 1,3 \\
2 (30\% aveia) & $0,99 \mathrm{a}, \mathrm{b}$ & $126,3 \mathrm{~b}$ & 19,7 & 1,0 \\
3 (45\% aveia) & $1,02 \mathrm{a}$ & $122,3 \mathrm{~b}$ & 18,7 & 0,6 \\
\hline
\end{tabular}

Médias seguidas pela mesma letra nas colunas não diferem entre si ao nível de $5 \%$ de significância pelo teste de Duncan.

Verificou-se diferença significativa entre as formulações com 15\%, 30\% e $45 \%$ de farinha de aveia quanto ao volume do produto pronto em comparação com a formulação com $0 \%$, sugerindo interferência da farinha de aveia na formação e expansão da estrutura protéica na massa. O volume do bolo resulta do uso de clara e suas proteínas, importantes na produção de produtos com baixa densidade e elevada expansibilidade em virtude da capacidade de incorporar ar e formar espumas (SGARBIERI, 1996). Os resultados obtidos sugerem que a composição química da farinha de aveia integral interferiu na formação e agregação da estrutura protéica ao redor das bolhas de ar na massa, contribuindo para a redução do volume do produto final. FOEGEDING, LUCK e DAVIS (2006) constataram que com a elevação da temperatura no forneamento, a desnaturação protéica e a gelatinização do amido determinam o volume do bolo, firmeza ou colapso de sua estrutura. 
Os resultados da avaliação de simetria variaram de $0 \mathrm{~mm}$ a $1,3 \mathrm{~mm}$, obtendo-se valores positivos para o contorno nos bolos com as diferentes formulações. O desenvolvimento simétrico e a qualidade de contorno de massa durante o forneamento constituem características importantes no processamento de produtos de panificação. Para bolos, a simetria ideal assume valor zero e o contorno deve ser positivo, significando crescimento uniforme da massa e manutenção estrutural durante a cocção.

A forma ou simetria de produtos de panificação deve ser uniforme e bem definida, caso contrário indica manuseio e processamento inadequados. Para KENT (1987) a simetria diferente de zero resulta, ainda, de massa muito dura com baixo teor de umidade, bem como fermentação inadequada, insuficiente ou manuseio incorreto e grosseiro.

\subsection{ANÁLISE SENSORIAL}

Verificou-se comportamento semelhante para a aceitação sensorial dos atributos sabor e textura (Tabela 4). Os bolos contendo 0\% e 30\% de farinha integral de aveia foram os mais aceitos pelos consumidores e não diferiram entre si $(p<0,05)$. Ambas as formulações obtiveram médias localizadas entre os termos "gostei moderadamente" e "gostei muito".

TABELA 4 - RESULTADOS DA ANÁLISE SENSORIAL DE ACEITAÇÃO PARA A AVALIAÇÃO DAS AMOSTRAS DE BOLO, CONSIDERANDO OS ATRIBUTOS SABOR, TEXTURA E IMPRESSÃO GLOBAL

\begin{tabular}{|c|c|c|c|c|}
\hline \multirow{2}{*}{ Atributos } & \multicolumn{4}{|c|}{ Formulações } \\
\hline & (0\%aveia) & 1 (15\%aveia) & 2 (30\%aveia) & 3 (45\%aveia) \\
\hline Sabor & $7,38 \mathrm{a}$ & $6,57 \mathrm{~b}$ & $7,21 a$ & $6,34 b$ \\
\hline Textura & $7,64 a$ & $6,45 b$ & $7,23 a$ & $6,53 b$ \\
\hline Impressão Global & $7,72 \mathrm{a}$ & $6,83 b, c$ & $7,21 a, b$ & $6,30 \mathrm{c}$ \\
\hline
\end{tabular}

Médias seguidas pela mesma letra nas linhas não diferem entre si ao nível de $5 \%$ de significância pelo teste de Duncan. 
Em termos de impressão global das amostras de bolos, a formulação contendo $0 \%$ de farinha integral de aveia diferiu significativamente $(p<0,05)$ das formulações com $15 \%$ e $45 \%$. No entanto, não foi constatada diferença significativa entre essa formulação e a contendo $30 \%$ de farinha integral de aveia. A maior aceitação do bolo contendo $0 \%$ de farinha integral de aveia pode ser explicada pelo fato dos consumidores que participaram do estudo estarem mais familiarizados com o emprego de farinha de trigo no preparo de bolos.

Todas as médias obtidas na aceitação sensorial localizam-se entre os pontos hedônicos 6 e 8, correspondentes aos termos "gostei ligeiramente" e "gostei muito".

\section{CONCLUSÃO}

A avaliação tecnológica das farinhas mistas, compostas por farinha integral de aveia e farinha de trigo, revelou características favoráveis à produção de bolos. As análises físicas demonstraram que a adição de farinha integral de aveia alterou significativamente a estrutura interna do produto final, confirmada pelo incremento nos valores de densidade da massa crua e redução do volume do produto. Embora as análises físicas não tenham evidenciado melhorias na aparência do produto, o bolo contendo $30 \%$ de farinha integral de aveia obteve aceitação similar ao bolo com $0 \%$ para os atributos sabor, textura e impressão global. Conclui-se que é viável a utilização dessa porcentagem de farinha de aveia para substituição parcial da farinha de trigo na elaboração de bolos.

\section{Abstract \\ USE OF OAT AND WHEAT COMPOSITE FLOUR IN CAKE MAKING}

The present work evaluated the technological and sensorial feasibility of the addition of oatmeal in wheat flour $(0,15,30$ and $45 \%$ addition) for cake production. Compared to wheat flour, oatmeal shows larger particle size, indicated by the higher particle retention when higher proportion of oatmeal was incorporated in the composite flour. Results from Farinograph showed that both water absorption and the time necessary to obtain a homogeneous paste increased with the increment of the oatmeal in the mixture. Cake batter density increased with the addition of oatmeal, which imparted a decrease on cake volume. Sensorial analysis revealed that formulation containing 0 and $30 \%$ of oatmeal were best accepted by consumers. The addition of $30 \%$ oatmeal did not seem to impart any significant alteration on 
flavor, texture and global impression of cakes. According to these results, it seems feasible to add $30 \%$ oatmeal in wheat flour for cake making.

KEY-WORDS: OATMEAL; CAKE; COMPOSITE FLOUR; WHEAT FLOUR.

\section{REFERÊNCIA}

1 AACC. American Association of Cereal Chemists. Approved methods of the AACC. $8^{\text {th }}$ ed. St. Paul, 1983. v. 1.

2 AACC. American Association of Cereal Chemists. Approved methods of the AACC. $10^{\text {th }}$ ed. St. Paul, 2000.

3 ANVISA. Agência Nacional de Vigilância Sanitária de Alimentos. Portaria no 354, de 18 de julho de 1996. Norma técnica referente à farinha de trigo. Disponível em: http://www.anvisa.gov.br/ anvisalegis/portarias/354_96.htm. Acesso em 20 abril 2006.

4 ANZALDÚA-MORALES, A. La evaluación sensorial de los alimentos en la teoría y la práctica. Zaragoza, Espanha: Editorial Acribia, 1994. $198 \mathrm{p}$.

5 BARBOSA, M. C. A. Avaliação tecnológica de massas alimentícias de farinha mista de trigo e soja sem lipoxigenases. Viçosa, 2002. 100 p. Tese (Doutorado em Ciência e Tecnologia de Alimentos), Universidade Federal de Viçosa (UFV).

6 BORGES, J. T. S.; ASCHERI; J. L. R.; ASCHERI, D. R.; NASCIMENTO, R. E.; FREITAS, A. S. Propriedades de cozimento e caracterização físico-química de macarrão pré-cozido à base de farinha integral de quinoa (Chenopodium quinoa, Wild) e de farinha de arroz (Oryza sativa, L) polido por extrusão termoplástica. Boletim CEPPA, Curitiba, v. 21, n. 2, p. 303-322, jul./dez. 2003.

7 EL-DASH, A.; GERMANI, R. Tecnologia de farinhas mistas: uso de farinhas mistas na produção de bolos. Brasília: EMBRAPASPI, 1994. v. 7. 31 p.

8 EL-DASH, A. A.; CAMARGO C. R. O. Fundamentos da tecnologia de panificação. São Paulo: Secretaria da Indústria, Comércio e Tecnologia, 1982. 400 p.

9 FOEGEDING, E. A.; LUCK, P. J.; DAVIS, J. P. Factors determing the physical properties of protein foams. Food Hydrocolloids, v. 20, 
p. 284-292, 2006.

10 GRIZARD, D.; DALLE, M.; BARTHOMEUF, C. Changes in insulin and corticosterone levels may partly mediate the hypolipidemic effect of guar gum and low-molecular weight pectin in rats. Nutritional Researches, v. 21, n. 8, p. 1185-1190, Aug. 2001.

11 GUTKOSKI, L, C.; TROMBETTA, C. Avaliação dos teores de fibra alimentar e de beta-glicanas em cultivares de aveia (Avena sativa L.). Ciência e Tecnologia de Alimentos, Campinas, v. 19, n. 3, p. $387-390,1999$.

12 GUTKOSKI, L.C.; PEDÓ, I. Aveia: composição química, valor nutricional e processamento. São Paulo: Varela, 2000. 96 p.

13 GUTKOSKI, L. C.; JACOBSEN NETO, R. Procedure to laboratorial test of bread making: form bread type. Ciência Rural, v. 32, n. 5, p. 873-879, set./out. 2002.

14 GUTKOSKI, L. C.; VELLOSO, C. B.; DÓRO, C. T.; SILVEIRA, A. A. E.; BONAFÉ, L. Z. Uso de farinha mista de trigo e aveia em produtos de panificação: pães tipo forma, francês e pré-pizza. Boletim do CEPPA, Curitiba, v. 11, n. 1, p. 33-45, 1993.

15 HOSENEY, R. C. Principles of cereal science and technology. St. Paul: American Association of Cereal Chemists, 1990. p. 76-87.

16 IDRIS, N.; ENBONG, M. S.; ABDULLCH, A.; CHEHA, C. M.; HASSAR, $H$. Performance evaluation of shortenings based on palm oil and butterfat in yellow cake. Fett/Lipid, Kuala Lumpur, v. 98, n. 4, p. $144-148,1996$.

17 IWUOHA, C. I.; NWAKANMA, M. I. Density and viscosity of cold flour pastes of cassava (Manihot esculenta Grantz), sweet potato (Ipomoea batatas L. Lam.) and white yam (Dioscorea Rotundata Poir) tuber as affected by concentration and particle size. Carbohydrate Polymers, v. 37, p. 97-101, 1998.

18 KENT, N. L. Tecnologia de los cereales. Zaragoza (Espanha): Acríbia, 1987.

19 LINDEN, G.; LORIENT, D. Bioquimica agroindustrial. España (zaragoza): Acribia, 1994. 426 p.

LOCKART, V. B.; HURT, D. Nutrition of oats. In: WEBSTER, F. H. Oats: chemistry and technology. St. Paul, Minessota: American 
Association of Cereal Chemists, 1986. p. 297-308.

21

MORR, C. V.; HOFFMANN, W.; BUCHHEIM, W. Use of applied air pressure to improve the baking properties of whey protein isolates in angel food cakes. Lebensmittell-Wissenschaft undTechnologie, v. 36, n. 1, p. 83-90, 2003.

MOSCATTO, J. A.; PRUDENCIO-FERREIRA, S. H.; HAULY, M. C. O. Farinha de yacon e inulina como ingredientes na formulação de bolo de chocolate. Ciência e Tecnologia de Alimentos, v. 24, n. 4, p. 634-640, out./dez. 2004.

OTT, R. L. An introduction to statistical methods and data analysis. $4^{\text {th }}$ ed. Belmont, CA: Duxbury Press, 1993. $1049 \mathrm{p}$.

PATON, D.; LAROCQUE, G. M.; HOLME, J. Development of cake structure: influence of ingredients on the measurement of cohesive force during baking. Cereal Chemistry, Saint Paul, v. 58, n. 6, p. 527-529, 1981.

PEDÓ, I.; SGARBIERI, V. C. Caracterização química de cultivares de aveia (Avena sativa L.). Ciência e Tecnologia de Alimentos, Campinas, v. 17, n. 2, p. 78-83, 1997.

PETERSON, M. P. Composition and nutritional characteristics of oat grain and product. In: MARSHALL, H. G.; SOLLELS, M. S. Oat science and technology. Madison: American Society of Agronomy, 1992. p. 266-287.

PYLER, E. J. Baking: science \& tecnology. $3^{\text {rd }}$ ed. Kansas: Sosland Publishing Company, 1988. v. 2.

QUALGLIA, G. Ciencia y tecnología de la panificación. 2. ed. España (Zaragoza): Acribia, 1991. 485 p.

ROY, S.; VEJA-LOPEZ, S.; FERNANDEZ, M. L. Gender and hormonal status affect the hypolipidemic mechanisms of dietary soluble fiber in guinea pigs. Journal of Nutrition, v. 130, n. 3, p. 600-607, Mar. 2000.

SÁ, R. M.; DE FRANCISCO, A.; OGLIARI, P. J.; BERTOLDI, F. C. Variação no conteúdo de beta-glucanas em cultivares brasileiros de aveia. Ciência e Tecnologia de Alimentos, Campinas, v. 20, n. 1, p. 99-102, 2000.

SAS Institute Inc. SAS user's guide: statistics. $5^{\text {th }}$ ed. Cary NC, 
1985. 965 p.

32 SEABRA, L. M. J.; ZAPATA, J. F. F.; NOGUEIRA, C. M.; DANTAS, M. A.; ALMEIDA, R. B. Fécula de mandioca e farinha de aveia como substitutos de gordura na formulação de hambúrguer de carne ovina. Ciência e Tecnologia de Alimentos, Campinas, v. 22, n. 3, p. 245-248, set./dez. 2002.

33 SGARBIERI, V. C. Proteínas em alimentos protéicos: propriedades, degradação e modificações. São Paulo: Varela, 1996. 520 p.

34 STONE, H.; SIDEL, J. L. Sensory evaluation practices. $2^{\text {nd }}$ ed. Redwood City: Academic Press, 1993. 338 p.

35 TEDRUS, G. A. S.; ORMENESE, R. C. S. C.; SPERANZA, S. M. Effects of the addition of vital gluten to rice flour, oat flour and wheat starch on the quality of breads. Ciência Tecnologia Alimentos, v. 21, n. 1, p. 20-25, jan./abr. 2001.

36 TEDRUS, G. A. S.; ORMENESE, R. C. S. C.; SPERANZA, S. M.; CHANG, Y. K.; BUSTOS, F. M. Estudo da adição de vital glúten à farinha de arroz, farinha de aveia e amido de trigo na qualidade de pães. Ciência e Tecnologia de Alimentos, Campinas, v. 21, n. 1, p. 20-25, jan./abr. 2001.

37 TIBURCIO, D.T.S. Enriquecimento protéico de farinha de madioca com farinha de soja de sabor melhorado: desenvolvimento e avaliação nutricional de um novo produto. Viçosa, 2000. 67 p. Dissertação (Mestrado em Ciência e Tecnologia de Alimentos), Universidade Federal de Viçosa.

38 WEBER, F. H.; GUTKOSKI, L. C.; ELIAS, M. C. Chemical characterization oat caryopses of the UPF 18 cultivar. Ciência e Tecnologia de Alimentos, v. 22, n. 1, p. 39-44, jan./abr. 2002.

39 WEBSTER, F. H. Oat utilization: past, present, and future. In: WEBSTER, F. H. Oats chemistry and technology. Saint Paul: American Association of Cereal Chemists, 1986. p. 413-426.

40 WOOD, P. J.; WEIZ, J.; BLACKWELL, B. A. Molecular characterization of cereal $\beta$-D-glucans. Structural analysis of oat B-D-glucan and rapid structural evaluation of $\beta$-D-glucans from different sources by high-performance liquid chromatography of oligosaccharides released by lichenase. Cereal Chemistry, Saint Paul, v. 68, n. 1, p. 31-39, 1991. 